\title{
Kaon photoproduction from the deuteron in a Regge-plus-resonance approach
}

\author{
P. Vancraeyveld, L. De Cruz, J. Ryckebusch*, T. Vrancx \\ Department of Physics and Astronomy, Ghent University, Proeftuinstraat 86, B-9000 Gent, \\ Belgium
}

\begin{abstract}
We present a Regge-inspired effective-Lagrangian framework for kaon photoproduction from the deuteron. Quasi-free kaon production is investigated using the Regge-plus-resonance (RPR) elementary operator within the relativistic planewave impulse approximation. The RPR model was developed to describe photoinduced and electroinduced charged-kaon production off protons. We show how this elementary operator can be transformed in order to account for the production of neutral kaons from both protons and neutrons. The model results for kaon photoproduction from the deuteron compare favourably to the ${ }^{2} \mathrm{H}(\gamma, K) Y N$ data published to date.

Keywords: Kaon production, Regge phenomenology, Baryon resonances
\end{abstract}

\section{Introduction}

Electromagnetic production of strangeness plays a prominent role in the quest to chart the excitation spectrum of the nucleon. Since the production mechanism inevitably involves quark-antiquark components of the nucleon's sea, the reaction has the potential to probe unexplored aspects of the nucleon's structure.

Leading experimental facilities have contributed to a large database for the

\footnotetext{
* Corresponding author

Email addresses: pieter.vancraeyveld@ugent.be (P. Vancraeyveld), jan.ryckebusch@ugent.be (J. Ryckebusch)
}

Preprint submitted to Elsevier

October 23, 2012 
$p\left(\gamma, K^{+}\right) \Lambda$ and $p\left(\gamma, K^{+}\right) \Sigma^{0}$ observables [1, 2]. The self-analysing weak decay of hyperons $Y$ is an enormous asset, since it facilitates the determination of the recoil polarisation. Hence, a wide range of single- and double-polarisation observables can be accessed by combining a polarised beam and/or target. This paves the way for the determination of a complete set of observables.

In addition to the study of $p\left(\gamma, K^{+}\right) Y$, it pays to consider strangeness production on more complex targets, such as the deuteron. First, owing to the deuteron's weak binding, it is ideally suited as an effective neutron target and gives access to the elementary $n(\gamma, K) Y$ reaction process. Second, by comparing reactions off free and bound protons, our understanding of nuclear-medium effects is put to the test. An important source of medium effects are the rescatterings between the hyperon, nucleon and kaon. These can be considered as an undesirable background effect when exploiting the deuteron as a neutron target, as it obscures the physics at the photon-neutron-kaon vertex. On the other hand, the final-state interactions (FSI) provide us with a tool to improve our understanding of the hyperon-nucleon $(Y N)$ and kaon-nucleon $(K N)$ potentials. Focusing on kinematic regions with major hyperon rescatterings allows one to gain access to the elusive $Y N$ interaction.

This letter investigates semi-inclusive strangeness production from the deuteron within the relativistic plane-wave impulse approximation (RPWIA). The next section introduces the Regge-plus-resonance (RPR) formalism for modelling elementary strangeness production. Section 3 focuses on how to embed the RPR production operator in the nuclear medium. In Section 4, we present model calculations and compare them to data. Here, the sensitivity of the results to the model's assumptions is investigated. Finally, we present our conclusions and indicate directions for future work.

\section{Elementary kaon production}

A relatively high production threshold and the absence of plain resonant structures in the energy dependence of the measured cross section point towards 
a dominance of non-resonant contributions to electromagnetic kaon production. This sets strangeness production apart from reactions such as $N(\gamma, \pi) N$ and $N\left(\pi, \pi^{\prime}\right) N^{\prime}$, and calls for a unique formalism that addresses the $N(\gamma, K) Y$ peculiarities. The RPR approach seeks to decouple the determination of the coupling constants for the background and the resonant diagrams. This results in a hybrid model which accounts for electromagnetic kaon production from threshold up to $E_{\gamma}=16 \mathrm{GeV}$. The generic structure of the transition current operator in the RPR approach reads

$$
\begin{aligned}
\hat{\mathbf{J}}_{K Y}= & \hat{\mathbf{J}}_{\text {Regge }}^{K^{+}{ }_{(494)}} \\
& +\hat{\mathbf{J}}_{\text {Regge }}^{K^{*+}(892)} \\
& +\hat{\mathbf{J}}_{\text {Feyn }}^{p, \text { elec }} \times \mathcal{P}_{\text {Regge }}^{K^{+}(494)} \times\left(t-m_{K^{+}}^{2}\right) \\
& +\sum_{N^{*}} \hat{\mathbf{J}}_{\text {Feyn }}^{N^{*}}+\sum_{\Delta^{*}} \hat{\mathbf{J}}_{\mathrm{Feyn}}^{\Delta^{*}} .
\end{aligned}
$$

The non-resonant contributions of Eqs. 1a and (1b) are efficiently modelled in terms of $t$-channel $K^{+}(494)$ and $K^{*+}(892)$ Regge-trajectory exchange $[3$. The three coupling constants can be determined from the high-energy $\left(E_{\gamma} \gtrsim 4 \mathrm{GeV}\right)$ data 4. A crucial constraint is gauge invariance. The $t$-channel Born diagram of Eq. 1a by itself is not gauge invariant. Adding the electric part of a Reggeized $s$-channel Born diagram (1c) ensures that the $p\left(\gamma, K^{+}\right) Y$ amplitude is gauge invariant [3. The Regge amplitudes are supplemented with $s$-channel nucleon $\left(N^{*}\right)$ and delta $\left(\Delta^{*}\right)$ resonance-exchange diagrams $(1 \mathrm{~d})$, whose parameters are optimised to data in the resonance-region $\left(E_{\gamma} \lesssim 4 \mathrm{GeV}\right)$ while keeping the background anchored.

High-quality kaon-photoproduction data over an extended energy and angular range is only available for $p\left(\gamma, K^{+}\right) \Lambda$ and $p\left(\gamma, K^{+}\right) \Sigma^{0}$. An economical description of these reactions has been obtained [5] and will be referred to as RPR-200打 For $p\left(\gamma, K^{+}\right) \Lambda$, a set of established nucleon resonances turns

\footnotetext{
${ }^{1}$ The RPR-2007 model corresponds to the $p\left(\gamma, K^{+}\right) \Lambda$ model labelled 'RPR-2 $+D_{13}(1900)$ ' and the $p\left(\gamma, K^{+}\right) \Sigma^{0}$ model labelled as 'RPR-3' ' in Table 1 of Ref. [7. The relevant coupling constants are listed in Appendix I of Ref. [9].
} 
out to be insufficient. The addition of a $D_{13}(1900)$ resonance makes it possible to accurately describe both photo- and electroproduction data [5, 7]. The $p\left(\gamma^{(*)}, K^{+}\right) \Sigma^{0}$ data, on the other hand, is properly described within the RPR framework considering established $N^{*}$ 's and $\Delta^{*}$ 's [6, 7].

A total of six $N(\gamma, K) Y$ reactions can be treated within a single theoretical framework. The two $\Lambda$ and the four $\Sigma$ reactions can be described by a single set of parameters based on the RPR-2007 model which is optimised to $p\left(\gamma, K^{+}\right) \Lambda$ and $p\left(\gamma, K^{+}\right) \Sigma^{0}$ data. The reaction channels can be interrelated by converting the coupling constants which feature in the interaction Lagrangians while maintaining gauge invariance. For $K^{0} \Lambda$ and $K^{0} \Sigma^{0}$ production, the kaon-exchange amplitude (1a) vanishes and gauge-invariance restoration becomes irrelevant. Further, $n\left(\gamma, K^{+}\right) \Sigma^{-}$is the only channel with a neutron target and a charged kaon. Accordingly, the electric part of the $s$-channel Born diagram $11 \mathrm{c}$ is identically zero. A gauge-invariant amplitude is obtained by including the electric part of a Reggeized $u$-channel Born diagram.

In the strong-interaction vertex, one can fall back on $\mathrm{SU}(2)$ isospin symmetry to find the relevant conversion factors, since the hadronic couplings are proportional to the Clebsch-Gordan coefficients [8, 9]. We adopt the isospin conventions of Ref. [8]. The strong coupling constants for the $\Lambda$-production channels are isospin independent. The strong-interaction vertices for $p\left(\gamma, K^{0}\right) \Sigma^{+}$can be related to those for $p\left(\gamma, K^{+}\right) \Sigma^{0}$

$$
\begin{aligned}
g_{K^{(*) 0} \Sigma^{+} p} & =\sqrt{2} g_{K^{(*)+} \Sigma^{0} p}, \\
g_{K^{(*) 0} \Sigma^{+} N^{*+}} & =\sqrt{2} g_{K^{(*)+} \Sigma^{0} N^{*+}}, \\
g_{K^{(*) 0} \Sigma^{+} \Delta^{*+}} & =\frac{-1}{\sqrt{2}} g_{K^{(*)+} \Sigma^{0} \Delta^{*+}} .
\end{aligned}
$$

Similar expressions apply for $n\left(\gamma, K^{+}\right) \Sigma^{-}$

$$
\begin{aligned}
g_{K^{(*)+\Sigma^{-} n}} & =\sqrt{2} g_{K^{(*)+} \Sigma^{0} p}, \\
g_{K^{(*)+} \Sigma^{-} N^{* 0}} & =\sqrt{2} g_{K^{(*)+} \Sigma^{0} N^{*+}}, \\
g_{K^{(*)+\Sigma^{-}} \Delta^{* 0}} & =\frac{1}{\sqrt{2}} g_{K^{(*)+} \Sigma^{0} \Delta^{*+}} .
\end{aligned}
$$


The transformation of the $p\left(\gamma, K^{+}\right) \Sigma^{0}$ amplitude to the $n\left(\gamma, K^{0}\right) \Sigma^{0}$ one, requires sign changes

$$
\begin{aligned}
& g_{K^{(*) 0} \Sigma^{0} n}=-g_{K^{(*)+\Sigma^{0} p}}, \\
& g_{K^{(*) 0} \Sigma^{0} N^{* 0}}=-g_{K^{(*)}+\Sigma^{0} N^{*+}}, \\
& g_{K^{(*) 0} \Sigma^{0} \Delta^{* 0}}=g_{K^{(*)+} \Sigma^{0} \Delta^{*+}} .
\end{aligned}
$$

Unlike the coupling constants in the strong-interaction vertex, the transformation of those in the electromagnetic-interaction vertex cannot proceed without experimental input. We first focus on reactions with a neutron target and summarise some issues discussed in more detail in Ref. [8].

The partial decay width for the radiative decay of a resonance to the groundstate nucleon can be expressed in terms of photocoupling helicity amplitudes $\mathcal{A}_{J}^{N}$ which can be linked with current matrix elements. The latter can, for example, be calculated within a quark model [10], or with phenomenological interaction Lagrangians. Thereby, the $N^{*}\left(\kappa_{N^{*} N}^{(1,2)}\right)$ and $\Delta^{*}\left(\kappa_{\Delta^{*} N}^{(1,2)}\right)$ transition moments can be related to the $\mathcal{A}_{J}^{N}$. Inverting these relations and neglecting the small proton-neutron mass difference, we find 8

$$
\frac{\kappa_{N^{*} n}}{\kappa_{N^{*} p}}=\frac{\mathcal{A}_{1 / 2}^{n}}{\mathcal{A}_{1 / 2}^{p}}
$$

for spin- $1 / 2$ resonances, and

$$
\begin{aligned}
\frac{\kappa_{N^{*} n}^{(1)}}{\kappa_{N^{*} p}^{(1)}} & =\frac{\sqrt{3} \mathcal{A}_{1 / 2}^{n} \pm \mathcal{A}_{3 / 2}^{n}}{\sqrt{3} \mathcal{A}_{1 / 2}^{p} \pm \mathcal{A}_{3 / 2}^{p}}, \\
\frac{\kappa_{N^{*} n}^{(2)}}{\kappa_{N^{*} p}^{(2)}} & =\frac{\sqrt{3} \mathcal{A}_{1 / 2}^{n}-\frac{m_{p}}{m_{N^{*}}} \mathcal{A}_{3 / 2}^{n}}{\sqrt{3} \mathcal{A}_{1 / 2}^{p}-\frac{m_{p}}{m_{N^{*}}} \mathcal{A}_{3 / 2}^{p}},
\end{aligned}
$$

for spin-3/2 resonances.

As motivated in Ref. [8], we employ helicity amplitudes of the SAID analysis SM95 [1]. Table 1 lists the conversion factors for the resonances relevant to RPR-2007. Obviously the ratios have considerable error bars. Moreover, no information for the $D_{13}(1900)$ and $P_{13}(1900)$ is available. Therefore, we allow the ratios of the magnetic transition moments, $\kappa_{N^{*} n}^{(1,2)} / \kappa_{N^{*} p}^{(1,2)}$, to vary between -2 
Table 1: The ratio of the electromagnetic coupling constants to proton and neutron for selected nucleon resonances obtained with Eq. 3. The listed values are obtained using photocoupling helicity amplitudes from SAID analysis SM95 [1]. No experimental information exists for resonances of mass $1900 \mathrm{MeV}$, therefore we consider a broad range.

\begin{tabular}{cccc}
\hline Resonance & $\frac{\kappa_{N^{*} n}}{\kappa_{N^{*} p}}$ & $\frac{\kappa_{N^{*} n}^{(1)}}{\kappa_{N^{*} p}^{(1)}}$ & $\frac{\kappa_{N^{*} n}^{(2)}}{\kappa_{N^{*} p}^{(2)}}$ \\
\hline$S_{11}(1650)$ & $-0.22 \pm 0.07$ & - & - \\
$P_{11}(1710)$ & $-0.29 \pm 2.23$ & - & - \\
$P_{13}(1720)$ & - & $-0.38 \pm 2.00$ & $-0.50 \pm 1.08$ \\
$D_{13}(1900)$ & - & $0.00 \pm 2.00$ & $0.00 \pm 2.00$ \\
$P_{13}(1900)$ & - & $0.00 \pm 2.00$ & $0.00 \pm 2.00$ \\
\hline
\end{tabular}

and +2 . Since the transition strengths for $\Delta^{*} \rightarrow n \gamma, p \gamma$ are identical, we include the $D_{33}(1700), S_{31}(1900), P_{31}(1910)$, and $P_{33}(1920)$ with the electromagnetic coupling constants determined from $p\left(\gamma, K^{+}\right) \Sigma^{0}$.

We evaluate the predictive power of RPR-2007 for kaon photoproduction from the neutron using the two available $n\left(\gamma, K^{+}\right) \Sigma^{-}$data sets. The LEPS results 12 comprise differential cross sections (d.c.s.) and photon-beam asymmetries for $\cos \theta_{K}^{*} \geq 0.65$ and $1.5 \mathrm{GeV} \leq E_{\gamma} \leq 2.4 \mathrm{GeV}$. Recently, the CLAS collaboration has published a large set of $n\left(\gamma, K^{+}\right) \Sigma^{-}$d.c.s. [13]. These data cover incident photons from $0.8 \mathrm{GeV}$ to $3.6 \mathrm{GeV}$ on a liquid-deuterium target.

In Fig. 1. model predictions are set against CLAS results at four values for $E_{\gamma}$. At the highest energies, where resonance exchange has nearly died out, the d.c.s. peaks at forward angles. The exponential decrease of the d.c.s. as a function of $\cos \theta_{K}^{*}$ is distinctive for Regge-trajectory exchange. This characteristic feature of the $n\left(\gamma, K^{+}\right) \Sigma^{-}$d.c.s. disappears as one moves into the resonance region. The quality of agreement of the three-parameter Regge model is fair as the shape of the cross section is reproduced, yet its strength is overestimated. One observes destructive interference between the Reggeized background and the resonances. This effect considerably improves the overall agreement with the d.c.s. data, except at the lowest photon energy where the backward strength 


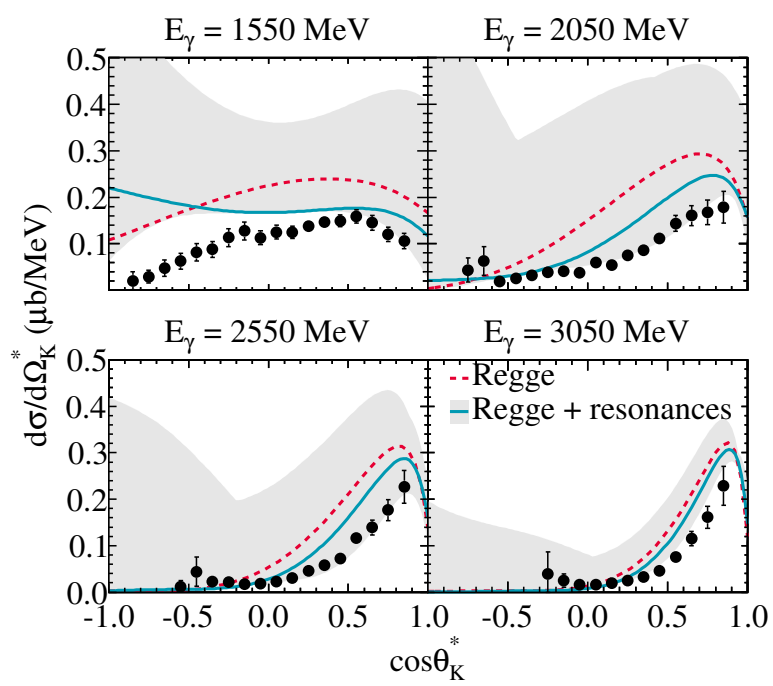

Figure 1: The $n\left(\gamma, K^{+}\right) \Sigma^{-}$differential cross section as a function of the kaon scattering angle $\cos \theta_{K}^{*}$ for 4 different values of the photon laboratory energy $E_{\gamma}$. The dashed curve indicates the contribution of the Reggeized background, whereas the full curve corresponds to the complete RPR result, i.e. background and resonance contributions. The shaded area takes the uncertainties of the helicity amplitudes from Table 1 into account. Data are from Ref. [13]. 
is overpredicted.

The $N^{*}$ coupling constants, listed in Table 1, have considerable error bars which induce uncertainties. Their impact is assessed in Fig. 1, and turns out to be quite dramatic. The shaded area indicates the range of values obtained for $d \sigma / d \Omega$ when the conversion factors of Table 1 are varied within their error bars. The experimental ambiguities of the transformed photon couplings result in deviations up to $100 \%$ for the d.c.s. Our results show that the resonant and Reggeized background amplitudes interfere destructively. This destructive interference is affected by varying the electromagnetic coupling constants of the resonances within their allowed ranges. With a large conversion factor, a particular resonance dominates and the cross section is enlarged. Hence, the error band in Fig. 1 is not positioned symmetrically around the central RPR prediction.

When transforming the electromagnetic vertex for $N\left(\gamma, K^{0}\right) Y$, the relevant coupling constants for $t$-channel $K^{+}(494)$ and $K^{*+}(892)$ exchange are the charge of the kaon and the magnetic transition moment $\kappa_{K^{*} K}$. In the transformation from $p\left(\gamma, K^{+}\right) Y^{0}$ to $N\left(\gamma, K^{0}\right) Y$, the electromagnetic vertices of the resonanceexchange terms $(1 \mathrm{~d})$ are unaffected.

In the $N\left(\gamma, K^{0}\right) Y$ channels, the contributions from the kaon-exchange diagram (1a) and the accompanying gauge-invariance-restoring $s$-channel diagram $1 \mathrm{c}$ vanish. Therefore, the $K^{* 0}(892)$-exchange diagram $1 \mathrm{~b}$ is the sole non-resonant contribution that survives. The decay width of $K^{*}$ vector meson to the ground-state kaon can be directly linked to the square of the magnetic transition moment. Adopting the decay widths listed in the Review of Particle Physics [14, one finds

$$
\frac{\kappa_{K^{* 0}(892) K^{0}(494)}}{\kappa_{K^{*+}(892) K^{+}(494)}}=-1.53 \pm 0.10 .
$$

The relative sign for these coupling constants cannot be deduced from experiment. We adopt the sign predicted by the quark models of Singer and Miller [15] and the Bonn group [16].

The $p\left(\gamma, K^{0}\right) \Sigma^{+}$reaction is the only neutral-kaon production channel where 
data is available. The use of Eqs. (2) and (4) implies that the $p\left(\gamma, K^{0}\right) \Sigma^{+}$yield will be roughly 4.7 times larger than the $p\left(\gamma, K^{+}\right) \Sigma^{0}$ one.

The data [6, 9], however, indicate that the $p\left(\gamma, K^{0}\right) \Sigma^{+}$yield is about $50 \%$ of the $p\left(\gamma, K^{+}\right) \Sigma^{0}$ yield near threshold. The absence of $K^{0} \Sigma^{+}$data at highenergies makes it impossible to constrain possible additional contributions to the Regge amplitude [9]. Therefore, we adopt a pragmatic approach, and fit the electromagnetic coupling constant of the $K^{*}(892)$ Regge trajectory to the available $p\left(\gamma, K^{0}\right) \Sigma^{+}$data to find [9]

$$
\frac{\kappa_{K^{* 0}(892) K^{0}(494)}}{\kappa_{K^{*+}(892) K^{+}(494)}}=0.054 \pm 0.010 .
$$

All other parameters in RPR-2007 are kept fixed. Despite the fact that only one free parameter is introduced, we attain $\chi^{2} / N_{\text {data }}=3.39$. With the fitted ratio of Eq. (5) the $K^{*}(892)$ trajectory is strongly suppressed and resonance exchanges play a more dominant role in $p\left(\gamma, K^{0}\right) \Sigma^{+}$.

\section{Modelling the ${ }^{2} \mathbf{H}(\gamma, K) Y N$ reaction}

Having established a model for all $N(\gamma, K) Y$ channels, they can now be embedded in the nuclear medium. The dominant contribution to ${ }^{2} \mathrm{H}(\gamma, K) Y N$ stems from the quasi-free process: the photon interacts with a single bound nucleon and produces the strange meson and hyperon. All final-state particles subsequently leave the interaction region as plane waves. All of them, however, can undergo elastic and inelastic rescatterings before reaching the detectors. These FSI can be considered a curse. Yet, FSI also present unique opportunities to access the $Y N$ interaction.

Yamamura et al. pioneered ${ }^{2} \mathrm{H}(\gamma, K) Y N$ investigations with a modern elementary kaon-production operator [17, 18, and included the $Y N$ FSI with the Nijmegen $Y N$ potential. The approach was extended [19] to include two-step production and kaon-nucleon rescattering. Adopting this model, neutral-kaon photoproduction has been studied. Refs. 20, 21] focus on the extraction of the

elementary amplitude. A different study on the influence of $Y N$ rescattering using the $P$-matrix approach is presented in Ref. [22]. Maxwell considered a host 
of rescattering diagrams with $\pi, \eta$ and $K$ exchanges between the active and the spectator nucleon [23, 24]. In Refs. [25, 26, ${ }^{2} \mathrm{H}(\gamma, K) Y N$ is investigated in the non-relativistic plane-wave impulse approximation (NRPWIA) with various isobar models, demonstrating the importance of a reliable elementary-production operator. Gasparyan et al. studied the possibility of extracting the low-energy $\Lambda n$ scattering parameters [27. Laget identified well-defined regions in phase space where $K N$ and $Y N$ rescattering dominate while the elementary amplitude is on shell and the momentum of the spectator nucleon is low [28, 29].

In our notation, the four-vectors of the photon, deuteron, kaon, hyperon and nucleon are $\boldsymbol{p}_{\gamma}\left(E_{\gamma}, \vec{p}_{\gamma}\right), \boldsymbol{p}_{D}\left(E_{D}, \vec{p}_{D}\right), \boldsymbol{p}_{K}\left(E_{K}, \vec{p}_{K}\right), \boldsymbol{p}_{Y}\left(E_{Y}, \vec{p}_{Y}\right)$ and $\boldsymbol{p}_{N}\left(E_{N}, \vec{p}_{N}\right)$. In what follows, all variables will be expressed in the laboratory frame, unless stated differently.

For the three-body final state, one can define a large number of coordinate systems. We label the final state as $1+(23)$. The coordinate system $(x, y, z)$, in which we describe the reaction, has its $z$ axis along $\vec{p}_{\gamma}$. The $y$ axis is chosen perpendicular to the plane spanned by the photon and particle 1 .

In order to improve on the statistics, the ${ }^{2} \mathrm{H}(\gamma, K) Y N$ data are often presented as semi-inclusive observables. Thereby, one integrates over the phase space of one or two final particles. We find for the semi-inclusive unpolarised d.c.s.

$$
\begin{aligned}
\frac{d^{3} \sigma_{\text {unpol }}}{d\left|\vec{p}_{1}\right| d \Omega_{1}}=\frac{1}{32(2 \pi)^{5}} \frac{\left|\vec{p}_{1}\right|^{2}\left|\vec{p}_{2}^{*}\right|}{m_{D} E_{\gamma} E_{1} W_{23}} & \\
& \times \frac{1}{3} \int d \Omega_{2}^{*} \sum_{\lambda_{D} \lambda_{N} \lambda_{Y}}\left|\mathcal{T}_{\lambda_{N}, \lambda_{Y}}^{\lambda_{D}, \lambda_{\gamma}=+1}\right|^{2} .
\end{aligned}
$$

Variables marked with an $*$ are evaluated in the c.m. frame of particles 2 and 3. Upon detecting the polarisation of the hyperon, a recoil polarisation asymmetry $\Pi_{y}$ can be defined $[9]$

$$
\begin{aligned}
& \Pi_{y} \frac{d^{3} \sigma_{\text {unpol }}}{d\left|\vec{p}_{Y}\right| d \Omega_{Y}}=\frac{1}{32(2 \pi)^{5}} \frac{\left|\vec{p}_{Y}\right|^{2}\left|\vec{p}_{N}^{*}\right|}{m_{D} E_{\gamma} E_{Y} W_{K N}} \\
& \quad \times \frac{1}{3} \int d \Omega_{N}^{*} \sum_{\lambda_{\gamma} \lambda_{D} \lambda_{N}} \Im\left[\left(\mathcal{T}_{\lambda_{N}, \lambda_{Y}=+\frac{1}{2}}^{\lambda_{D}, \lambda_{\gamma}}\right)^{*} \mathcal{T}_{\lambda_{N}, \lambda_{Y}=-\frac{1}{2}}^{\lambda_{D}, \lambda_{\gamma}}\right] .
\end{aligned}
$$


The dynamics of the reaction are contained in the transition amplitude $\mathcal{T}_{\lambda_{N}, \lambda_{Y}}^{\lambda_{D}, \lambda_{\gamma}}$, where $\lambda_{\gamma}, \lambda_{D}, \lambda_{Y}$ and $\lambda_{N}$ indicate the helicities, which are defined in the laboratory frame.

In this work, we adopt the impulse approximation, which states that the full nuclear many-body current operator can be approximated by a sum of one-body current operators. In the RPWIA, the Lorentz-invariant transition amplitude is given by

$$
\begin{aligned}
\mathcal{T}_{\lambda_{N}, \lambda_{Y}}^{\lambda_{D}, \lambda_{\gamma}}=\mp \sqrt{2} \bar{u}\left(\vec{p}_{Y}, \lambda_{Y}\right) & \epsilon_{\nu}^{\lambda_{\gamma}} \hat{\mathrm{J}}_{K Y}^{\nu} \\
& \times \frac{m_{T}+\boldsymbol{p}_{T}}{m_{T}^{2}-\left(\boldsymbol{p}_{T}\right)^{2}} \xi_{\mu}^{\lambda_{D}} \Gamma_{\mathrm{Dnp}}^{\mu}\left(\boldsymbol{p}_{N}, \boldsymbol{p}_{D}\right) \mathcal{C} \bar{u}^{T}\left(\vec{p}_{N}, \lambda_{N}\right),
\end{aligned}
$$

with $\mathcal{C}=-i \gamma^{0} \gamma^{2}$ the charge conjugation matrix. The mass and four-vector of the struck nucleon are given by $m_{T}$ and $\boldsymbol{p}_{T}$. The factor $\mp \sqrt{2}$ stems from isospin factors, and the fact that the production operator acts on a single proton (neutron) 9. Since the spectator nucleon is on mass shell, the covariant Dnp-vertex $\boldsymbol{\Gamma}_{\text {Dnp }}$ is defined by 30 .

$$
\begin{aligned}
\Gamma_{\mathrm{Dnp}}^{\mu}\left(\boldsymbol{p}_{N}, \boldsymbol{p}_{D}\right)=F(|\vec{p}|) \gamma^{\mu} & -\frac{G(|\vec{p}|)}{m_{N}} p^{\mu} \\
& -\frac{m_{N}-\left(\boldsymbol{p}_{D}-\boldsymbol{p}_{N}\right)}{m_{N}}\left(H(|\vec{p}|) \gamma^{\mu}-\frac{I(|\vec{p}|)}{m_{N}} p^{\mu}\right),
\end{aligned}
$$

with $\boldsymbol{p}=\frac{1}{2} \boldsymbol{p}_{D}-\boldsymbol{p}_{N}$. The four scalar form factors $F, G, H$ and $I$ can be expressed in terms of the $S$-, $P$ - and $D$-wave components of the deuteron wave function [9, 31].

The target nucleon is obviously off its mass shell and the $\hat{\mathbf{J}}_{K Y}$ of Eq. (8) is evaluated with one off-mass-shell leg. Owing to the deuteron's tiny binding energy, the virtuality is minor for small spectator-nucleon momenta $\vec{p}_{N}$. In order to assess the uncertainties induced by off-shell extrapolations, we introduce the on-shell four-vector $\widetilde{\boldsymbol{p}}_{T} \stackrel{\mathrm{LAB}}{\equiv}\left(\sqrt{\left|\vec{p}_{N}\right|^{2}+m_{T}^{2}},-\vec{p}_{N}\right)$ of the target nucleon. After decomposing the nucleon propagator in Eq. (8), we obtain for the on-shell part 
of the RPWIA amplitude [9]

$$
\begin{aligned}
\mathcal{T}_{\lambda_{N}, \lambda_{Y}}^{\lambda_{D}, \lambda_{\gamma}} \stackrel{\text { LAB }}{=} \mp \sqrt{2} \sqrt{(2 \pi)^{3} 2 m_{D}} \frac{2 E_{N}}{m_{D}} \\
\quad \times \sum_{\lambda_{T}}\left\langle\boldsymbol{p}_{K} ; \boldsymbol{p}_{Y}, \lambda_{Y}\left|\hat{J}_{K Y}^{\lambda_{\gamma}}\right| \widetilde{\boldsymbol{p}}_{T}, \lambda_{T}\right\rangle \Psi_{\lambda_{T} \lambda_{N}}^{++}\left(-\vec{p}_{N}, \lambda_{D}\right),
\end{aligned}
$$

with $\Psi^{++}$the positive-energy deuteron wave function. The above equation represents the NRPWIA expression for the transition amplitude, up to a kinematical factor $2 E_{N} / m_{D}$. The amplitude of Eq. 10 is evaluated with all incoming and outgoing particles on their mass shells. It needs to be evaluated in the laboratory frame unlike Eq. (8).

\section{Results}

The ${ }^{2} \mathrm{H}(\gamma, K) Y N$ results can be presented in terms of various combinations of the kinematic variables. For small outgoing nucleon momenta $\left(\left|\vec{p}_{N}\right| \ll m_{N}\right)$ and vanishing FSI the d.c.s. can be approximated by 9 ]

$$
\frac{d^{5} \sigma}{d \vec{p}_{N} d \Omega_{K}^{*}} \stackrel{\mathrm{LAB}}{\approx}\left(1+\frac{\left|\vec{p}_{N}\right|}{E_{N}} \cos \theta_{N}\right) \frac{\rho_{D}\left(\left|\vec{p}_{N}\right|\right)}{2 \pi} \frac{d^{2} \sigma_{K Y}}{d \Omega_{K}^{*}},
$$

with $\rho_{D}$ the deuteron density and $d^{2} \sigma_{K Y} / d \Omega_{K}^{*}$ the elementary $K Y$ cross section. Owing to the factorised form (11) of the d.c.s., known as the non-relativistic spectator-nucleon approximation 32, we can assess which regions in phase space are to contribute most to the reaction's strength. The elementary amplitude exhibits only mild variations with energy, whereas $\rho_{D}$ falls off exponentially with increasing $\left|\vec{p}_{N}\right|$. Under those conditions that the reaction is dominated by the RPWIA contribution, most strength will emerge in phase-space regions corresponding to low $\left|\vec{p}_{N}\right|$. This is confirmed in Fig. 2, where the semi-inclusive d.c.s. is shown as function of $\left|\vec{p}_{N}\right|$ and $\cos \theta_{N}$. We notice an exponential falloff as $\left|\vec{p}_{N}\right|$ increases, reminiscent of the deuteron density. The d.c.s. is nearly isotropic. The bands in Fig. 2 are only slightly tilted in the clockwise direction. This indicates a mild dependence on $\cos \theta_{N}$ that becomes stronger as the spectator momentum rises. 


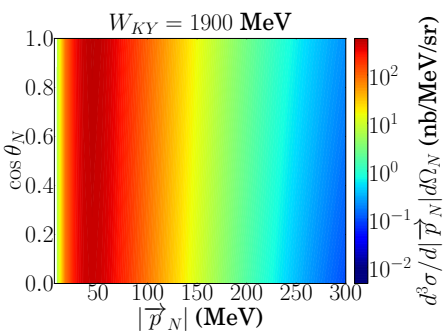

Figure 2: The semi-inclusive ${ }^{2} \mathrm{H}\left(\gamma, K^{+}\right) Y N$ differential cross section using the full RPR operator in the RPWIA as a function of the nucleon momentum $\left|\vec{p}_{N}\right|$ and scattering angle $\cos \theta_{N}$ in the $\mathrm{LAB}$ frame at $W_{K Y}=1900 \mathrm{MeV}$.

We investigate the sensitivity of the computed cross sections to the various model ingredients in Fig. 3 . The role of off-shell effects can be examined by comparing the RPWIA of Eq. (8) to its on-shell reduction (10). In the right panel of Fig. 3, we compare the one-fold ${ }^{2} \mathrm{H}(\gamma, n) K^{+} \Lambda$ d.c.s. as calculated with both forms of the transition amplitude. Clearly, the RPWIA result and the on-shell approximation almost coincide for $\left|\vec{p}_{N}\right| \lesssim 350 \mathrm{MeV}$. At large $\vec{p}_{N}$, the results bifurcate, with the on-shell form of the transition amplitude giving significantly larger cross sections than the RPWIA. Accordingly, in phase-space regions with small $\left|\vec{p}_{N}\right|$, off-shell ambiguities are absent, and one can extract information on the on-shell $n(\gamma, K) Y$ amplitude.

As illustrated earlier, the deuteron density shapes the ${ }^{2} \mathrm{H}(\gamma, K) Y N$ cross section. The left panel of Fig. 3 shows the $\left|\vec{p}_{N}\right|$ dependence of the ${ }^{2} \mathrm{H}(\gamma, n) K^{+} \Lambda$ d.c.s. for various deuteron wave functions. For $\left|\vec{p}_{N}\right| \lesssim 300 \mathrm{MeV}$ one obtains nearly indistinguishable results. This comes as no surprise, because all $N N$ potentials produce comparable ${ }^{3} S_{1}$ waves. As the spectator nucleon's momentum rises, the cross-section predictions start to diverge. The non-relativistic wave functions of the Paris 34] and Nijmegen [35] potentials and the relativistic Gross-IIB [36] wave function generate very similar predictions. The cross sections based on the CD-Bonn [33] and WJC-1 [37] potentials, on the other hand, differ up to an order of magnitude at high missing momenta.

To date, the only published ${ }^{2} \mathrm{H}(\gamma, K) Y N$ data are from the Laboratory 


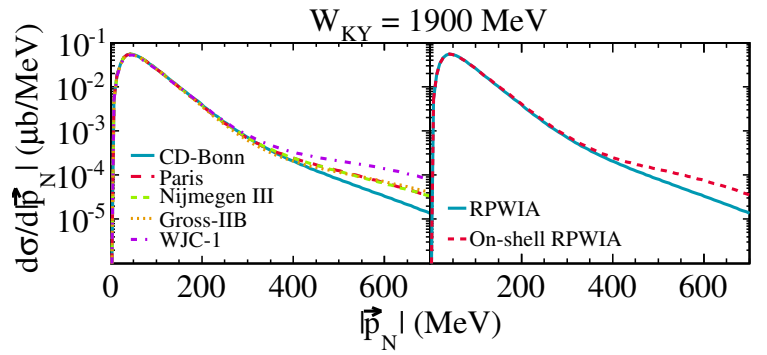

Figure 3: The ${ }^{2} \mathrm{H}(\gamma, n) K^{+} \Lambda$ differential cross section as a function of the nucleon momentum $\left|\vec{p}_{N}\right|$ at $W_{K Y}=1900 \mathrm{MeV}$. In the left panel, results are obtained within the RPWIA adopting different versions of the deuteron wave function: CD-Bonn [33, Paris 34, Nijmegen-III 35], Gross-IIB [36] and WJC-1 37. The right panel compares for the CD-Bonn wave function, the RPWIA result (solid curve) with the one obtained with the on-shell reduction of Eq. 10 (dashed curve).

for Nuclear Science (LNS) at Tohoku University [38, 39]. The semi-inclusive ${ }^{2} \mathrm{H}\left(\gamma, K^{0}\right) Y N$ cross sections have been measured in two $100 \mathrm{MeV}$-wide $E_{\gamma}$ bins close to threshold.

In Fig. 4, our RPWIA results are compared to the LNS data. The model calculations are performed at the centre of the $E_{\gamma}$ bin. As energies close to the $\Sigma$-production threshold are probed, the cross section has a uni-modal $\vec{p}_{K}$ distribution at $\left\langle E_{\gamma}\right\rangle=950 \mathrm{MeV}$, whereas a second structure arises for the $\left\langle E_{\gamma}\right\rangle=1050 \mathrm{MeV}$ bin. The RPWIA predictions reproduce the shape of the data and the characteristic quasi-elastic peaks of semi-inclusive kaon production. At $\left\langle E_{\gamma}\right\rangle=950 \mathrm{MeV}$ the strength is underpredicted by roughly a factor of two. The resonant contributions play an unsubstantial role. At $\left\langle E_{\gamma}\right\rangle=1050 \mathrm{MeV}$, the RPWIA reproduces the measured magnitude of the cross sections. The reaction is dominated by the Reggeized background but the $N^{\star}$ contributions are essential to reproduce the observed shoulder at $\left|\vec{p}_{K}\right| \approx 300 \mathrm{MeV}$, which corresponds to quasi-elastic $\Sigma$ production.

In Section 2, the error bars on experimental helicity amplitudes emerged as a chief source of theoretical uncertainties. Because both $K^{0} \Lambda$ and $K^{0} \Sigma^{0}$ production from the neutron contribute to ${ }^{2} \mathrm{H}\left(\gamma, K^{0}\right) Y N$, one can expect a 


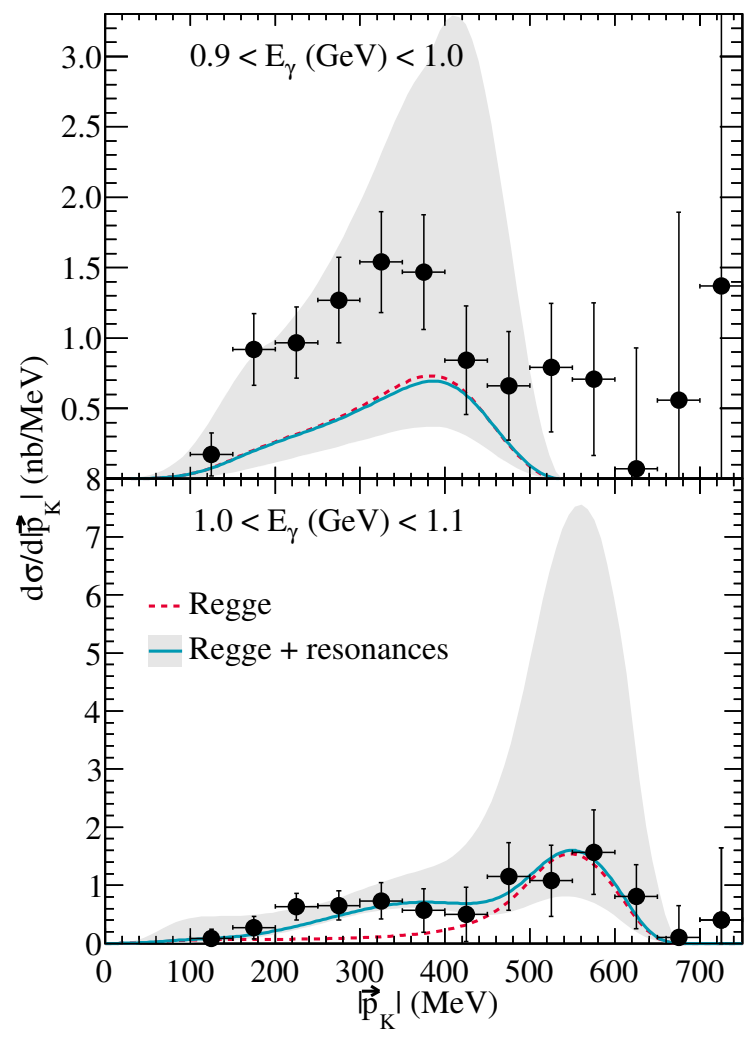

Figure 4: The semi-inclusive ${ }^{2} \mathrm{H}\left(\gamma, K^{0}\right) Y N$ differential cross section as a function of the kaon momentum $\left|\vec{p}_{K}\right|$ integrated over $0.9 \leq \cos \theta_{K} \leq 1$. The results in the upper (lower) panel have been calculated at $E_{\gamma}=950(1050) \mathrm{MeV}$. The solid (dashed) line shows the result using the full RPR (Reggeized background) amplitude. The shaded area shows the effect of the uncertainties on the adopted helicity amplitudes given in Table 1 The data are from Refs. 38, 39]. 


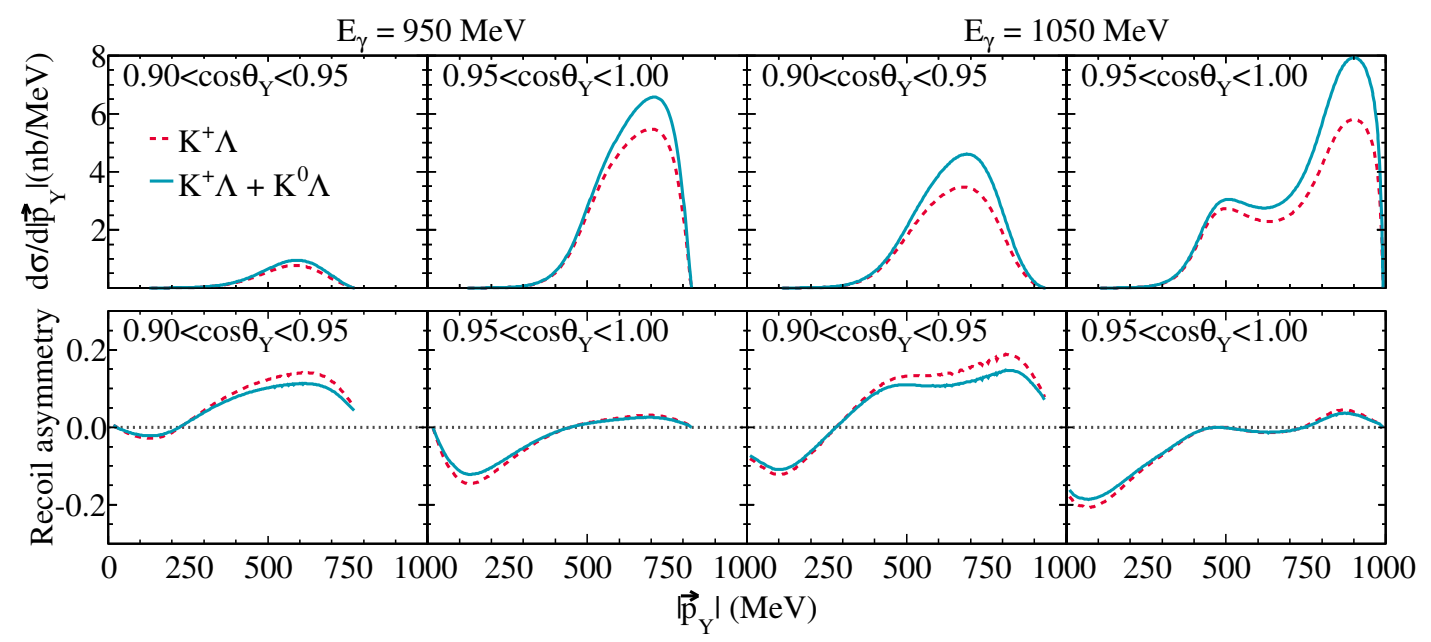

Figure 5: The semi-inclusive differential cross section (top row) and recoil asymmetry (bottom row) for ${ }^{2} \mathrm{H}(\gamma, \Lambda) K N$ as a function of the hyperon momentum $\left|\vec{p}_{Y}\right|$ integrated over the $\theta_{Y}$ bin marked in each panel. The results in the left (right) panels have been calculated at $E_{\gamma}=950(1050) \mathrm{MeV}$. The solid line shows the result using the RPR amplitude in the RPWIA, whereas the dashed curve singles out the ${ }^{2} \mathrm{H}(\gamma, \Lambda) K^{+} n$ contribution.

considerable impact. The shaded band in Fig. 4 represents the cross sections obtained with the range of coupling constants of Table 1 . At $\left\langle E_{\gamma}\right\rangle=950 \mathrm{MeV}$, where the RPR predictions with the central values of the coupling constants underestimate the data, the missing strength can be compensated by including the helicity-amplitude errors. The errors in the quasi-elastic $\Lambda$-production peak are large compared to those for $\Sigma$ production. This can be understood if one considers the resonant content of the RPR model for $\Lambda$ production. The resonances $P_{13}(1900)$ and $D_{13}(1900)$ play a role and their photon-helicity couplings have not been determined experimentally. This forced us to introduce sizable error bars on the ratios of their coupling constants.

In Ref. [38] it is argued that the shape of the kaon momentum spectra of the data presented in Fig. 4 is mainly determined by the angular distribution of the elementary kaon-production cross sections. The RPR-2007 model, which is used as elementary-production amplitude in this work, has been fitted to $K^{+} \Lambda$ and $K^{+} \Sigma^{0}$ production data at forward kaon angles. Therefore, for the 
deuteron calculations there is some uncertainty stemming from the contributions of backward kaon angles. We have verified, however, that the semi-inclusive ${ }^{2} \mathrm{H}\left(\gamma, K^{0}\right) Y N$ differential cross sections are dominated by the strength from forward angles.

In recent years, dedicated efforts to expand the ${ }^{2} \mathrm{H}(\gamma, K) Y N$ database have been undertaken. At LNS, new data have been collected with an upgraded spectrometer [40, 41]. Thereby, the hyperon polarisation becomes accessible [42]. In Fig. 5. RPR-model hyperon-momentum distributions are presented for the semi-inclusive cross section and recoil asymmetry at LNS kinematics. The shape of the cross section is determined by the momentum of the struck nucleon, and changes as a function of $E_{\gamma}$ and $\vec{p}_{Y}$. The reaction is dominated by $K^{+} \Lambda$ production. Our calculations suggest a moderate recoil asymmetry that changes sign as a function of $E_{\gamma}$ and $\left|\vec{p}_{Y}\right|$. For $\cos \theta_{Y} \in[0.95,1]$, the asymmetry is close to zero, except at low $\left|\vec{p}_{Y}\right|$ where the cross section nearly vanishes. For $\cos \theta_{Y} \in[0.9,0.95]$, the asymmetry is mildly negative at small $\left|\vec{p}_{Y}\right|$, and grows to $0.1-0.15$ at quasi-elastic kinematics. Here, the $K^{0} \Lambda$ channel has its largest effect, and reduces the size of the recoil asymmetry.

\section{Conclusions and outlook}

In the proposed RPR framework for strangeness photoproduction, the analysis of the resonant and non-resonant content of kaon production is effectively decoupled. We gauge the predictive power of the RPR-2007 model, whose parameters are constrained by $p\left(\gamma, K^{+}\right) Y^{0}$ data, and extend the formalism to reactions with a neutron target and/or a neutral kaon in the final state.

The RPR-2007 production operator is used in a covariant formalism to study ${ }^{2} \mathrm{H}(\gamma, K) Y N$ reactions. In the leading RPWIA contribution to the reaction amplitude, the momentum distribution of the deuteron emerges as the dominant factor that dictates the angular and momentum dependence of the cross sections. Uncertainties related to the deuteron wave function or off-shell effects are only important at large missing momenta. Hence, the elementary amplitude can 
be most readily determined based on data obtained at small spectator-nucleon momenta.

Model predictions for semi-inclusive $K^{0}$ photoproduction in the threshold region are compared to experimental results. All predictions compare favourably to the data. The incomplete knowledge of helicity amplitudes induces important uncertainties on the model calculations.

Anticipating new ${ }^{2} \mathrm{H}(\gamma, \Lambda) K N$ data, we presented predictions for semi-inclusive $\Lambda$-production cross sections and recoil asymmetries. In future work, the quality of our formalism will benefit from the new RPR-model analysis of the world's $p\left(\gamma, K^{+}\right) \Lambda$ data [43, 44. In addition, inclusion of the different rescattering contributions will allow to study exclusive kaon photoproduction at a more detailed level, and single out phase-space regions where the elusive hyperon-nucleon potential can be investigated.

\section{Acknowledgements}

This work was supported by the Research Foundation - Flanders (FWO) and the research council of Ghent University. The calculations were carried out using the Stevin Supercomputer Infrastructure at Ghent University, funded by Ghent University, the Hercules Foundation and the Flemish Government department EWI.

\section{References}

[1] E. Klempt, J.-M. Richard, Rev.Mod.Phys. 82 (2010) 1095.

[2] V. Burkert, T. Lee, Int.J.Mod.Phys. E13 (2004) 1035.

[3] M. Guidal, J. M. Laget, M. Vanderhaeghen, Nucl.Phys. A627 (1997) 645.

[4] L. De Cruz, D. G. Ireland, P. Vancraeyveld, J. Ryckebusch, Phys.Lett. B694 (2010) 33.

[5] T. Corthals, J. Ryckebusch, T. Van Cauteren, Phys.Rev. C73 (2006) 045207. 
[6] T. Corthals, D. G. Ireland, T. Van Cauteren, J. Ryckebusch, Phys.Rev. C75 (2007) 045204.

[7] T. Corthals, T. Van Cauteren, P. Vancraeyveld, J. Ryckebusch, D. G. Ireland, Phys.Lett. B656 (2007) 186.

[8] P. Vancraeyveld, L. De Cruz, J. Ryckebusch, T. Van Cauteren, Phys.Lett. B681 (2009) 428.

[9] P. Vancraeyveld, Ph.D. thesis, Ghent University, 2011. http://inwpent5.ugent.be/Publication/phd/phdpietervan.pdf.

[10] D. Merten, U. Löring, K. Kretzschmar, B. Metsch, H. R. Petry, Eur.Phys.J. A14 (2002) 477.

[11] R. A. Arndt, I. I. Strakovsky, R. L. Workman, Phys.Rev. C53 (1996) 430.

[12] H. Kohri, et al., Phys.Rev.Lett 97 (2006) 082003.

[13] S. Anefalos Pereira, et al., Phys.Lett. B688 (2010) 289.

[14] J. Beringer, et al., Phys.Rev. D86 (2012) 010001.

[15] P. Singer, G. A. Miller, Phys.Rev. D33 (1986) 141.

[16] T. Van Cauteren, J. Ryckebusch, B. Metsch, H.-R. Petry, Eur.Phys.J. A26 (2005) 339.

[17] H. Yamamura, K. Miyagawa, T. Mart, C. Bennhold, W. Gloeckle, Phys.Rev. C61 (2000) 014001.

[18] K. Miyagawa, T. Mart, C. Bennhold, W. Glöckle, Phys.Rev. C74 (2006) 034002.

[19] A. Salam, H. Arenhövel, Phys.Rev. C70 (2004) 044008.

[20] A. Salam, K. Miyagawa, T. Mart, C. Bennhold, W. Glöckle, Phys.Rev. C74 (2006) 044004. 
[21] A. Salam, T. Mart, K. Miyagawa, Mod.Phys.Lett. A24 (2009) 968.

[22] B. O. Kerbikov, Phys.Atom.Nucl. 64 (2001) 1835.

[23] O. V. Maxwell, Phys.Rev. C69 (2004) 034605.

[24] O. V. Maxwell, Phys.Rev. C70 (2004) 044612.

[25] P. Bydzovsky, Int.J.Mod.Phys. E19 (2010) 2369.

[26] P. Bydzovsky, M. Sotona, Nucl.Phys. A835 (2010) 246.

[27] A. Gasparyan, J. Haidenbauer, C. Hanhart, K. Miyagawa, Eur.Phys.J. A32 (2007) 61.

[28] J.-M. Laget, Phys.Rev. C73 (2006) 044003.

[29] J.-M. Laget, Phys.Rev. C75 (2007) 014002.

[30] R. Blankenbecler, L. F. Cook, Phys.Rev. 119 (1960) 1745.

[31] W. W. Buck, F. Gross, Phys.Rev. D20 (1979) 2361.

[32] J.-M. Laget, Phys.Rept. 69 (1981) 1-84.

[33] R. Machleidt, Phys.Rev. C63 (2001) 024001.

[34] M. Lacombe, et al., Phys.Rev. C21 (1980) 861.

[35] V. G. J. Stoks, R. A. M. Klomp, C. P. F. Terheggen, J. J. de Swart, Phys.Rev. C49 (1994) 2950.

[36] F. Gross, J. W. Van Orden, K. Holinde, Phys.Rev. C45 (1992) 2094-2132.

[37] F. Gross, A. Stadler, Phys.Rev. C78 (2008) 014005.

[38] K. Tsukada, et al., Phys.Rev. C78 (2008) 014001.

[39] K. Tsukada, et al., Phys.Rev. C83 (2011) 039904.

[40] B. Beckford, et al., AIP Conf.Proc. 1388 (2011) 280. 
[41] K. Futatsukawa, et al., EPJ Web Conf. 20 (2012) 02005.

[42] H. Kanda, et al., Nucl.Phys. A835 (2010) 317.

[43] L. De Cruz, T. Vrancx, P. Vancraeyveld, J. Ryckebusch, Phys.Rev.Lett. 108 (2012) 182002.

[44] L. De Cruz, J. Ryckebusch, T. Vrancx, P. Vancraeyveld, Phys.Rev. C86 (2012) 015212. 